\title{
Model analysis and simulation on impacts of COVID-19 pandemic on the economy: a case study of Thailand's GDP and its lock down
} measures

\author{
Chontita Rattanakul, Yongwimon Lenbury* \\ Received: October 28, 2020. Revised: November 10, 2020, Accepted: November 19, 2020. Published: November $20,2020$.
}

\begin{abstract}
COVID-19 could affect the global and local economy mainly by directly affecting production, by creation of disruption in supply chains and markets, as well as through its financial impact on firms and markets and organizations. However, the extent to which the impact is felt depends a great deal on the how governments and the public react to the disease. Here, a model is proposed to investigate the effect of the spread of corona virus infection and the consequent measures taken in response to its spread to lessen its impacts on the society and the economy. The interaction between the number of infected individuals and the variations in the national Growth Product, GDP, is modeled by a system of impulsive non-linear difference equations with delays. We are specifically interested in how different lock down measures effect business recovery as reflected by the national GDP. The model is analyzed to obtain valuable insights as to the factors that could yield different successes in the pandemic control and business recovery in various scenarios. Based on data of newly infected cases and cumulative cases weekly in Thailand, the model is simulated in a variety of scenarios to illustrate how different strategies and lockdown measures may give rise to different recovery rates.
\end{abstract}

Keywords- COVID-19, economic recovery, response measures, delayed impulsive system, difference equations, stability analysis.

\section{INTRODUCTION}

$\mathrm{A}$ ccording to [1], COVID-19 could affect the global economy in mainly three ways. Firstly, it exerts direct

This work was supported in part by the Centre of Excellence in Mathematics, Post Graduate Education and Research Development Office of the Ministry of Higher Education, Science, Research, and Innovation, Thailand, under Grant RG-01-(63-64)-01-1.

Chontita Rattanakul is with the Department of Mathematics, Faculty of Science, Mahidol University, Rama 6 Rd., Bangkok 10800, Thailand, and the Centre of Excellence in Mathematics, Post Graduate Education and Research Development Office of the Ministry of Higher Education, Science, Research, and Innovation, Thailand. (e-mail: chontita.rat@mahidol.ac.th).

Yongwimon Lenbury is with the Department of Mathematics, Faculty of Science, Mahidol University, Rama 6 Rd., Bangkok 10800, Thailand, and the Centre of Excellence in Mathematics, Post Graduate Education and Research Development Office of the Ministry of Higher Education, Science, Research, and Innovation, Thailand. (corresponding author, phone: (662) 201-5448; fax: 662-201-5335; e-mail: scylb@yahoo.com). impacts on production, as witnessed by what has occurred in China. It has become clear that Chinese production has already been considerably affected by the shutdown in Hubei province and other areas of the country. Some other countries have also to feel, to different levels, a direct impact as their governments impose similar measures. The slowdown in China has substantial effects on exporters to China, the largest sources of imports of which are Japan, Korea, and other Asian countries including Thailand, according to the World Bank [1]. For this reason, even discounting any possibility of new outbreaks of the disease, these areas will have a high chance of experiencing slow growth in the first half of 2020 , to say the least.

Secondly, as Bachman stated in [1], many manufacturing firms not only depend on intermediate inputs that are imported from China and other countries overwhelmed by the disease, they also rely on sales of their products in China to meet their goals of financial growth. Thus, the slowdown in business activity, exacerbated by transportation restrictions, in affected countries will most probably have an impact on companies globally in terms of their production and profitability, not to mention in the aspects of manufacturing and raw materials used in manufacturing [1]. If companies rely heavily on intermediate goods from affected regions, they may not be immediately capable of easily switching from one source to the next. The extent to which the companies are affected depends on how quickly the outbreak declines and how stringent the measures imposed by the authorities to mitigate the crisis. More importantly, businesses connected to travel and tourism are unlikely to recover from the losses that they have been facing.

Finally, it is also stated in [1] that the third channel through which the COVID-19 crisis could affect the global economy is by its financial impact on financial firms and markets. Lengthy disruptions of production will cause stress on some firms, specifically those with insufficient liquid assets. To correctly anticipate or understand which firms might be vulnerable, traders in financial markets face difficulties in not being able to correctly foresee future trends in these volatile times. The corresponding rise in risk will likely portend a significant decline in equity markets and corporate bond markets. 
As the coronavirus continues its spread around the globe, governments have turned to proven public health measures, such as social distancing, and mask wearing, to physically disrupt the disease [2]. However, doing so has significantly cut off the flow of goods and people as mentioned above, slow economies, and is undoubtedly creating a global recession. According to Carlsson-Szlezak et al. [2], "economic contagion is now spreading as fast as the disease itself."

As the virus began to spread initially, politicians, policy makers, and investors, aware of the pattern outbreaks in history, wasted valuable time by not acting in a timely fashion during which time early, and thus more effective and less costly, window for social distancing closed [2]. Now, it has become clear that the economic costs have reached a much higher level, since the pandemic trajectory has progressed much further along, so that forecasting the outcome has turned out to be next to impossible, as there have arisen many more unprecedented dimensions of the crisis which are unforeseen [2]. In this uncharted territory, the role of mathematical models has without question gained importance and credibility in their forecasting power.

As explained in [3], the majority of models can be classified into two categories, which are proposed in order to achieve different objectives: projections versus statistical forecasts. Projection models are deterministic and they predict what could happen given a set of underlying assumptions, while statistical forecasting models utilize observed data to predict what will happen in the future.

In 1927, Kermack and McKendrick [4] developed the famous continuous predictive model of epidemic population dynamics, known as a "susceptible, infected, and removed" (SIR) model which divided a constant population into three compartments or states. The first state represents individuals susceptible to the infection denoting by $S(t)$ the number of susceptible individuals on day $t$ into the epidemic. In the second state belongs the infected individuals on day $t$, denoted by $I(t)$. Finally, $R(t)$ denotes the number of individuals removed from the infectious disease dynamics through death or recovery with immunity on day $t$ of the epidemic.

It is assumed that susceptible individuals contract the infection by interacting with infected individuals. This interaction is modeled by a term which varies as the product of the susceptible and the infected, $r S(t) R(t)$. Moreover, the mortality/recovery rate is modeled as a direct proportion of infected individuals. The key contribution of such projection models like that of Kermack- McKendrick's is that they are based on logical assumptions of the underlying mechanism of a process so that they can be utilized to immediately address "what if" questions without having to rely on a great deal of supporting data [4].

More importantly, it is from SIR- like models that we can see the effects of social distancing on flattening the curve [3]. In addition, once the model parameters are fitted to experimental data, SIR models can predict when infections may peak or how high the peak may be, depending on the underlying model assumptions, as pointed out in Jewell et al. [5].
However, it is argued by many researchers in the statistical camp [3] that it is important that researchers use projection models for hypothetical scenario development and statistical forecasting models for forecasting, which are two different goals.

The average number of secondary cases because of one infected person at the beginning of the epidemic is denoted by $R_{0}$, and in order for the epidemic to decline $R_{0}$ should be less than 1. Within the SIR framework, $R_{0}=r N T$, where $T$ is the time to recover and $N$ is the population size. In absence of interventions, the estimated $R_{0}$ of COVID- 19 is between 1.5 and 6.7 [6]. This value is not constant but changing with time. According to [7], $R_{0}$ can be fit to a statistical model. Similarly to Massad et al. [8], Carlsson-Szlezak [3] fitted an exponential decay model to New York's data yielding $R_{0}=e^{0.5-0.02 t}$, though it is suggested that other statistical models such as one with an asymptote could be utilized.

Here, we propose a model to investigate the effect of the spread of corona virus infection and the consequent measures taken in response to its spread to lessen its impacts on the society and the economy. We utilize the national Growth Product (GDP) per capita as the variable that best reflects the economic health of a country. The interaction between the number of infected individuals and the variations in the national GDP per capita is modeled by a system of non-linear difference equations with impulses at variable intervals. The model tracks the number of mobile healthy individuals in the population, the number of infected individuals, and the variations in GDP per capita. The instances of national impositions of lock down restrictions and relaxations at different levels and intensities are incorporated on the model dynamics by the addition of impulses at variable intervals and strengths. We also take into account the delays before infected individuals show symptoms, as well as the delays before business can recover after the lock down has been lifted. The recovered individuals return to the mobile population after a delay as well. Thus, the model is a system of delayed impulsive difference equations. We are specifically interested in how different lock down measures effect business recovery as reflected by the national GDP per capita. The model is analyzed to obtain valuable insights as to the factors that could yield different successes in the pandemic control and business recovery in various scenarios. Based on data of newly infected cases and cumulative cases weekly in Thailand, the model is simulated in a variety of scenarios to illustrate how different scenarios of strategies and lockdown measures may give rise to different recovery rates.

\section{MODEL SYSTEM}

In order to model the impact of coronavirus pandemic on the economy and subsequent recovery, we let $h_{n}$ be the number, in millions, of healthy mobile individuals at week $n$ who are able to interact with others and carry out transactions within the community. We let $b_{n}$ denote the indicator of business health, taken here to be the national GDP per capita at week $n$, and let $i_{n}$ denote the number of newly infected 
individuals in the community at week $n$.

Based on the principles of the fundamental SIR model, we then arrive at the following system, where $k=1,2,3, \ldots$, $m=0,1,2, \ldots, T-d-1$, and $d$ is the delays before the stimuli from incoming visitors or workers on the business recovery is felt as reflected by the increase in GDP.

$h_{n+1}=h_{n}-r_{i} h_{n-1} i_{n-1}+c h_{n}, n \neq k T$,

$b_{n+1}=b_{n}+\sigma\left(h_{n}+i_{n-3}\right)^{2}-L b_{n}+\kappa b_{n} h_{n}, n \neq(2 k-1) T+m+d$,

$i_{n+1}=i_{n}+r_{i} h_{n-1} i_{n-1}, n \neq(2 k-1) T+m+d$,

$h_{n+1}=\left\{\begin{array}{l}\left(1+q_{u k}\right) h_{n}, n=(2 k-1) T \\ \left(1-q_{d k}\right) h_{n}, n=2 k T\end{array}\right.$,

$b_{n+1}=(1+\beta) b_{n}, n=(2 k-1) T+m+d$,

$i_{n+1}=i_{n}+v, n=(2 k-1) T+m+d$.

Equation (1) gives the number of mobile healthy individuals at week $n+1$ in terms of that at week $n$. The second term on the right of (1) is the rate of removal of healthy mobile individuals due to infection by the infected population $i_{n}$ so that this term, as in the SIR model, is taken to be directly proportional to the product of $h_{n-l}$ and $i_{n-l}, r_{i}$ being the removal rate constant of variation. We assume that an infected individual takes an average of 2 weeks before being detected, and hence the subscript $n-1$ on $h$ and $n$ in this term. The third term here represents the changes in the population due to natural causes, taken to directly vary as the population level at time $n$ with variation constant $c, 0<c<1$.

Equation (2) gives the value of GDP per capita at time $n+1$ in terms of that at time $n$. The second term on the right of (2), with the proportional constant $L, 0<L<1$, is the rate of increase of GDP due to stimulation from interaction among mobile individuals, which includes the recovered individuals who take on the average 4 weeks to recover and hence the subscript $n-3$ here. The last 2 terms in (2) represent the rate of business loss which decreases at the rate $\kappa b_{n} h_{n}$ with the increase in the interaction between $h_{n}$ and $b_{n}$.

Equation (3) gives the number of infected individuals at time $n+1$ as a sum of that at time $n$ and the number of newly infected individuals $r_{i} h_{n-1} i_{n-1}$ with the delay of 2 weeks.

However, equation (1) applies only in the weeks where $n \neq k T$ because this is the duration where there is no impositions of lockdown measures or their relaxations. If $n=(2 k-1) T$, equation (4) applies, at which instance the lockdown is lifted with $q_{u k}$ being the fraction of $h_{n}$ that are allowed to interact socially with others in the community. If $n=2 k T$ on the other hand, equation (4) will also apply at which instance a lockdown is enforced with $q_{d k}$ being the fraction of $h_{n}$ that are kept from interacting socially with others in the community. Thus, $T$ is the length of the periods of lockdown and lockdown relaxation.

$$
\text { Equations (2) and (3) apply only when }
$$
$n \neq(2 k-1) T+m+d, \quad k=1,2,3, \ldots, \quad m=0,1,2, \ldots, T-d-1$, during which time no business stimulus from inbound travelers is felt. At the times $n, n=(2 k-1) T+m+d, k=1,2,3, \ldots$, $m=0,1,2, \ldots, T-d-1$, the stimuli from inbound visitors or workers will kick in as reflected by the GDP that jumps up by a fraction of $\beta b_{n}$ as in (5), and infectious population feels weekly jumps of size $v$ from infected individuals from outside the country as in (6), after a delay of $d$ weeks.

The parameters in this model system (1) - (6) are varied to simulate different lockdown and relaxation measures in various scenarios in Section IV. We first carry out an analysis of the model in the next section.

\section{MODEL ANALYSIS}

Here, we carry out a stability analysis of (1) - (6) by first equating their right hand sides to zero to find 2 equilibrium solutions as follows.

1) The trivial steady state solution:

$$
(h, b, i)=\left(h_{0}, b_{0}, i_{0}\right) \equiv(0,0,0),
$$

a washout steady state at which all state variables vanish. Healthy as well as infected human become extinct, in which case no business can exist.

2) The endemic equilibrium solution:

$$
(h, b, i)=\left(h_{s}, b_{s}, i_{s}\right)=\left(0, \frac{\sigma c^{2}}{L r_{i}^{2}}, \frac{c}{r_{i}}\right),
$$

at which point healthy population becomes extinct while infection is endemic.

We next consider each of the steady states individually by writing

$$
h_{n}=C \lambda^{n},
$$

so that

$$
h_{n-1}=C \lambda^{n-1}=h_{n} \lambda^{-1} .
$$

\section{A. The Washout Steady State}

We linearize the model system about the steady state $\left(h_{0}, b_{0}, i_{0}\right) \equiv(0,0,0)$ to derive the following The Jacobian matrix:

$$
J(0,0,0)=\left(\begin{array}{ccc}
1+c & 0 & 0 \\
0 & 1-L & 0 \\
0 & 0 & 1
\end{array}\right),
$$

whose characteristic equation is

$$
(1-\lambda)(1+c-\lambda)(1-L-\lambda)=0,
$$

yielding the eigenvalues

$$
\lambda_{1}=1+c, \lambda_{2}=1-L, \lambda_{3}=1 .
$$

Since $c>0, \lambda_{1}>1$ and therefore, the washout steady state is unstable.

\section{B. The Endemic Steady State}

Linearizing the model system (1) - (6) about the point $\left(h_{s}, b_{s}, i_{s}\right)=\left(0, \frac{\sigma c^{2}}{L}, \frac{c}{r_{i}}\right)$, we arrive at the following Jacobian matrix. 


$$
J\left(h_{s}, b_{s}, i_{s}\right)=\left(\begin{array}{ccc}
1-r_{i} \lambda^{-1} i_{s}+c & 0 & 0 \\
2 \sigma i_{s} \lambda^{-3}+\kappa b_{s} & 1-L & 2 \sigma i_{s} \lambda^{-3} \\
r_{i} i_{s} \lambda^{-1} & 0 & 1
\end{array}\right) .
$$

Thus, the characteristic equation is

$$
(1-\lambda)\left(1-r_{i} \lambda^{-1} i_{s}+c-\lambda\right)(1-L-\lambda)=0,
$$

yielding the eigenvalues

$$
\lambda_{1}=1, \lambda_{2}=1-L, \lambda_{3}=c .
$$

Thus, $\left|\lambda_{1,2,3}\right| \leq 1$.

The eigenvectors corresponding to the above 3 eigenvalues are

$$
\bar{e}_{1}=\left(\begin{array}{c}
0 \\
\frac{2 \sigma i_{s}}{L} \\
1
\end{array}\right), \bar{e}_{2}=\left(\begin{array}{l}
0 \\
1 \\
0
\end{array}\right) \text {, and } \bar{e}_{3}\left(\begin{array}{c}
1 \\
m_{s} \\
l
\end{array}\right)
$$

where

$$
m_{s}=\frac{\left(2 \sigma i_{s}+\kappa b_{s}\right)(1-c) c^{2}+2 c \sigma i_{s}}{c[c+(L-1)]}, l=\frac{c}{c-1} .
$$

Letting

$$
P=\left(\begin{array}{ccc}
1 & 0 & 0 \\
m_{s} & 1 & \frac{2 \sigma i_{s}}{L} \\
l & 0 & 1
\end{array}\right)
$$

and

$$
L(n)=\left(\begin{array}{ccc}
c^{n} & 0 & 0 \\
0 & (1-L)^{n} & 0 \\
0 & 0 & 1
\end{array}\right),
$$

then, the fundamental solution matrix of (1) - (3) is given by

$$
\begin{aligned}
& \phi(n)=P L(n)=\left(\begin{array}{ccc}
1 & 0 & 0 \\
m_{s} & 1 & \frac{2 \sigma i_{s}}{L} \\
l & 0 & 1
\end{array}\right)\left(\begin{array}{ccc}
c^{n} & 0 & 0 \\
0 & (1-L)^{n} & 0 \\
0 & 0 & 1
\end{array}\right) \\
& =\left(\begin{array}{ccc}
c^{n} & 0 & 0 \\
m_{s} c^{n} & (1-L)^{n} & \frac{2 \sigma i_{s}}{L} \\
l c^{n} & 0 & 1
\end{array}\right) .
\end{aligned}
$$

We consider this in 2 separate cases.

Case 1: the fraction of population put under lockdown equals that taken out of lockdown; $q_{u k}=q_{d k}=q_{k}$

Linearizing (4) - (6) at $(2 k-1) T$, we obtain the fundamental matrix

$$
E_{1}=\left(\begin{array}{ccc}
1+q_{k} & 0 & 0 \\
0 & 1 & 0 \\
0 & 0 & 1
\end{array}\right),
$$

which leads us to

$$
\Phi_{1}(T)=E_{1} \phi(T)=\left(\begin{array}{ccc}
\left(1+q_{k}\right) c^{T} & 0 & 0 \\
m_{s} c^{T} & (1-L)^{T} & \frac{2 \sigma i_{s}}{L} \\
l c^{T} & 0 & 1
\end{array}\right) .
$$

Since there is the delay of $d$, we first find the matrix of transition during the period of $d$ as

$$
\Phi_{2}(d)=\left(\begin{array}{ccc}
c^{d} & 0 & 0 \\
m_{s} c^{d} & (1-L)^{d} & \frac{2 \sigma i_{s}}{L} \\
l c^{d} & 0 & 1
\end{array}\right) .
$$

Then, by linearizing (4) - (6) during the corresponding interval after the delay, we find the fundamental matrix

$$
E_{2}=\left(\begin{array}{ccc}
1 & 0 & 0 \\
0 & (1+\beta) & 0 \\
0 & 0 & 1
\end{array}\right),
$$

which leads us to

$$
\Phi_{3}(T)=E_{2} \phi(1)=\left(\begin{array}{ccc}
c & 0 & 0 \\
m_{s} c(1+\beta) & (1+\beta)(1-L) & \frac{2 \sigma i_{s}(1+\beta)}{L} \\
l c & 0 & 1
\end{array}\right),
$$

for every week during the period of $T-d$ weeks. Linearizing (4) - (6) at $2 k T$, we obtain

$$
\Phi_{4}(T)=\left(\begin{array}{ccc}
1-q_{k} & 0 & 0 \\
0 & 1 & 0 \\
0 & 0 & 1
\end{array}\right) .
$$

Thus, the stability of the solution $\left(h_{s}, b_{s}, i_{s}\right)=\left(0, \frac{\sigma c^{2}}{L r_{i}^{2}}, \frac{c}{r_{i}}\right)$ is determined by the eigenvalues of

$$
\Phi_{4} \Phi_{3}^{T-d} \Phi_{2} \Phi_{1}=\left(\begin{array}{ccc}
c^{2 T}\left(1-q_{k}^{2}\right) & 0 & 0 \\
* & (1-L)^{2 T}(1+\beta)^{T-d} & * \\
* & 0 & 1
\end{array}\right),
$$

where the entries shown in the above matrix as $*$ are not relevant to our conclusions and hence need not be derived.

We are thus able to prove the following theorem.

Theorem 1: For the model system (1) - (6), the endemic steady state $\quad\left(h_{s}, b_{s}, i_{s}\right)=\left(0, \frac{\sigma c^{2}}{L}, \frac{c}{r_{i}}\right) \quad$ will be stable, but not asymptotically stable, provided 


$$
\left|1-q_{k}^{2}\right| \leq 1
$$

and

$$
T \leq \frac{d \ln (1+\beta)}{(\ln (1+\beta)+2 \ln (1-L))}
$$

hold. If either (10) or (11) is violated, then the steady state will be unstable.

\section{Proof}

Considering (9), the eigenvalues of $\Phi_{4} \Phi_{3}^{T-d} \Phi_{2} \Phi_{1}$ are

$$
\begin{aligned}
& \lambda_{1}=\left(1-q_{k}^{2}\right) c^{2 T}, \\
& \lambda_{2}=(1+\beta)^{T-d}(1-L)^{2 T}, \text { and } \\
& \lambda_{3}=1 .
\end{aligned}
$$

Since (10) holds, $\left|\lambda_{1}\right| \leq 1$, Since (11) holds, we will have $\left|\lambda_{2}\right| \leq 1$. Finally, $\left|\lambda_{3}\right|=1$ and therefore, the steady state is stable.

On the other hand, if either (10) or (11) is violated, one of the eigenvalues has absolute value greater than 1 which means the steady state is unstable.

Case 2: fraction of population put under lockdown different from that taken out of lockdown; $q_{u k} \neq q_{d k}$.

In this case, if we write

$$
q_{u k}=q_{d k}+\Delta, \Delta>0,
$$

then we have the following result.

Theorem 2: If (12) holds, and

$$
\frac{1}{1-q_{d k}}>\left(1+q_{d k}\right) \text {, }
$$

then, the endemic steady state $\left(h_{s}, b_{s}, i_{s}\right)$ is stable provided

$$
\Delta \leq \frac{1}{1-q_{d k}}-\left(1+q_{d k}\right) .
$$

On the other hand, $\left(h_{s}, b_{s}, i_{s}\right)$ will be unstable if

$$
\Delta>\frac{1}{1-q_{d k}}-\left(1+q_{d k}\right) \text {. }
$$

\section{Proof}

The reference matrix in this case will be similar to that of Case 1 given in (9) but with the entry in the first row and first column being

$$
\lambda_{1}=\left(1+q_{u k}\right)\left(1-q_{d k}\right) c^{2 T}
$$

instead. Thus, recalling the $0<c<1$, we have stability when this quantity is less than or equal to 1 , and if this is greater than 1 , the steady state will be unstable.

Now, if (14) holds, then by rearranging, we obtain

$$
\left|\lambda_{1}\right|=\left(1+q_{u k}\right)\left(1-q_{d k}\right) c^{2 T}<\left(1+q_{d k}+\Delta\right)\left(1-q_{d k}\right) \leq 1 .
$$

which ensures stability. Instability follows with the sign reversed which makes this eigenvalue bigger than 1 .

We note that asymptotic stability cannot be assured because $\lambda_{3}=1$.

\section{INCREMENTAL OPENING UP OF LOCKDOWN}

We now modify the system of equations (1) - (6) to model the case in which relaxation of a lockdown is carried out incrementally. In other words, the businesses are allowed out of lockdown in a stepwise fashion. The modified model is as follows.

$$
\begin{aligned}
& h_{n+1}=h_{n}-r_{i} h_{n-1} i_{n-1}+c h_{n}, n \neq k T+m, m=1,2,3 \ldots, T-1, \\
& b_{n+1}=b_{n}+\sigma\left(h_{n}+i_{n-3}\right)^{2}-L b_{n}+\kappa b_{n} h_{n}, n \neq(2 k-1) T+m+d \text {, } \\
& m=0,1,2, \ldots, T-d-1,(17) \\
& i_{n+1}=i_{n}+r_{i} h_{n-1} i_{n-1}, n \neq(2 k-1) T+m+d, \\
& m=0,1,2, \ldots, T-d-1,(18) \\
& h_{n+1}=\left\{\begin{array}{l}
\left(1+q_{u k}\right) h_{n}, n=(2 k-1) T+m, m=0,2,3 \ldots, T-1, \\
\left(1-q_{d k}\right) h_{n}, n=2 k T
\end{array},\right. \\
& b_{n+1}=(1+\beta) b_{n}, n=(2 k-1) T+m+d, m=0,1,2, \ldots, T-d-1,(20) \\
& i_{n+1}=i_{n}+v, n=(2 k-1) T+m+d, m=0,1,2, \ldots, T-d-1 \text {, }
\end{aligned}
$$

where $k=1,2,3, \ldots$.

Here, the index $m$ allows $h_{n}$ to experience a jump every week during the period from $(2 k-1) T$ to $2 k T-1$, and then the cycle starts again at $2 k T$.

Noting that the system (16) - (21) has the same steady states as (1) - (6), we can prove the following result in a similar manner to the non-incremental strategy.

Theorem 3:

The endemic steady state $\left(h_{s}, b_{s}, i_{s}\right)$ of the model system (16) (21) is stable, but not asymptotically stable, provided

$$
T \leq T_{m},
$$

where

$$
T_{m}=\min \left\{\frac{\ln \left(1-q_{d k}\right)^{-1}}{\ln \left(1+q_{u k}\right)}, \frac{d \ln (1+\beta)}{(\ln (1+\beta)+2 \ln (1-L))}\right\} .
$$

It will be unstable if

$$
T>T_{m} \text {. }
$$

Proof

Similar to the proof of Theorem 2, linearization of (16) - (18) yields the fundamental matrix

$$
\Psi_{1}(n)=\left(\begin{array}{ccc}
c^{n} & 0 & 0 \\
m_{s} c^{n} & (1-L)^{n} & \frac{2 \sigma i_{s}}{L} \\
l c^{n} & 0 & 1
\end{array}\right) .
$$

For every week during the first $d$ weeks of the period between $(2 k+1) T$ and $2 k T, k=1,2,3, \ldots$, the healthy mobile population takes an upward jump of $q_{u k}$ so that we have the matrix

$$
\Psi_{2}=\left(\begin{array}{ccc}
1+q_{u k} & 0 & 0 \\
0 & 1 & 0 \\
0 & 0 & 1
\end{array}\right)\left(\begin{array}{ccc}
c^{T} & 0 & 0 \\
m_{s} c^{T} & (1-L) & \frac{2 \sigma i_{s}}{L} \\
l c^{T} & 0 & 1
\end{array}\right) .
$$


Then, during the remaining $T-d$ weeks, the matrix corresponding to business recovery together with human returns is

$$
\Psi_{3}=\left(\begin{array}{ccc}
1+q_{u k} & 0 & 0 \\
0 & (1+\beta) & 0 \\
0 & 0 & 1
\end{array}\right)\left(\begin{array}{ccc}
c^{T} & 0 & 0 \\
m_{s} c^{T} & (1-L) & \frac{2 \sigma i_{s}}{L} \\
l c^{T} & 0 & 1
\end{array}\right) .
$$

Finally, during the lockdown period, we have

$$
\Psi_{4}=\left(\begin{array}{ccc}
1-q_{d k} & 0 & 0 \\
0 & 1 & 0 \\
0 & 0 & 1
\end{array}\right)\left(\begin{array}{ccc}
c^{T} & 0 & 0 \\
m_{s} c^{T} & (1-L)^{T} & \frac{2 \sigma i_{s}}{L} \\
l c^{T} & 0 & 1
\end{array}\right) .
$$

The stability of the steady state of interest then depends on the sizes of the eigenvalues of

$$
\begin{aligned}
& \Psi_{4} \Psi_{3}^{T-d} \Psi_{2}^{d} \\
& =\left(\begin{array}{ccc}
\left(1-q_{d k}\right)\left(1+q_{u k}\right)^{T} c^{2 T} & 0 & 0 \\
* & (1+\beta)^{T-d}(1-L)^{2 T} & * \\
* & 0 & 1
\end{array}\right),
\end{aligned}
$$

where the expressions of off diagonal entries shown as * are not relevant to our conclusion.

If (22) holds, then

$$
T \leq \frac{1}{\ln \left(1+q_{u k}\right)} \ln \left(\frac{1}{1-q_{d k}}\right) .
$$

Rearranging (25), we see that the first eigenvalue

$$
\lambda_{1}=\left(1-q_{d k}\right)\left(1+q_{u k}\right)^{T} c^{2 T}<\left(1-q_{d k}\right)\left(1+q_{u k}\right)^{T} \leq 1,
$$

since $0<c<1$.

We also have

$$
T<\frac{d \ln (1+\beta)}{\ln (1+\beta)+2 \ln (1-L)},
$$

which makes, upon rearranging,

$$
\lambda_{2}=(1+\beta)^{T-d}(1-L)^{2 T} \leq 1 \text {. }
$$

Thus, if (22) holds, both (26) and (28) will be satisfied. On the other hand, if (24) holds, then one of the eigenvalues would be larger than 1 in size. Therefore, the steady state is stable if (22) holds and unstable if (24) does. It is not asymptotically stable since $\lambda_{3}=1$.

\section{RESULTS AND DISCUSSION}

\section{A. Parametric Values}

The values of the model parameters used in our simulations are given in Table 1. while the other parameters are left to be varied in order to illustrate different scenarios of infection control strategies adopted by the authority.
The value of $r_{i}$ has been deduced from the data of officially reported newly infected cases each week during March 29 to April 29, 2020, in Thailand [9], so that model yields a forecast that is the closest to the actual reported reported case of 42 new cases during the week from April 30, 2020 and May 6, 2020, which is used as the initial week of our simulations. With these parameter values, the model then predicts the numbers of new cases for the next 2 weeks after the initial week to be 17.4 and 21.37 cases which are very close to the reported new cases of 17 and 21, respectively [9].

According to [10], the current annual population growth rate is $0.25 \%$. Thus, we deduce the growth rate $c$ per week to be

$$
c=\frac{\text { annual growth rate }}{\text { number of weeks in a year }}=\frac{0.25}{52}=0.0048 \text { per week. }
$$

Table 1. Model Parameter Values and Units

\begin{tabular}{|c|c|c|}
\hline Parameter & Value & Unit \\
\hline$r_{i}$ & $1.6444 \times 10^{-5}$ & wk $^{-1}$ per mil. \\
\hline$c$ & 0.0048 & wk $^{-1}$ \\
\hline$\sigma$ & $0.033 \times 10^{-2}$ & wk $^{-1}$ per mil. \\
\hline$L$ & 0.00663 & wk $^{-1}$ \\
\hline$\kappa$ & 0.00078 & wk $^{-1}$ per mil. \\
\hline$T$ & 9 & weeks \\
\hline$v$ & 0.00025 & mil. \\
\hline
\end{tabular}

According to [11], Thailand's GDP per capita was $\$ 7,792$ in $2019,9 \%$ to $17.7 \%$ of which is due to tourism and related businesses [12]. The Bank of Thailand expects Thailand's GDP to be reduced by 5.3\% due to the COVID_19 crises [13]. Relying on these numbers, The variation constant in the term that represents business stimulation due to human interaction $\sigma$ has been estimated to be

$$
\begin{aligned}
\sigma & \cong \frac{\text { predicted GDP drop during lockdown }}{\text { fraction mobile } \times \text { total population } \times \text { weeks lockdown }} \\
& =\frac{5.3 \%}{0.25 \times 69.8 \times 9 \mathrm{wks}} \cong 0.033 \% \text { per million persons per week } .
\end{aligned}
$$

The value of $L$ in rate of business/opportunity loss at reduced mobile human is accordingly estimated as

$$
\begin{aligned}
L \cong & \frac{\text { predicted GDP drop }}{\text { number of weeks to acheive such drop in GDP }} \\
& \cong \frac{5.3 \%}{8 \text { weeks }}=0.00663 \text { per million persons, }
\end{aligned}
$$

assuming that it takes around 8 weeks for businesses $t$ fully recover the loss due to COVID-19 situation in Thailand.

$k$ is the parameter of effect of mobile human contributing to increase in business growth rate:

$$
\kappa=\frac{1.923 \text { per capita }}{52 \text { wks } \times 0.679 \times 69.8 \text { million }}=0.00078 \text {. }
$$

Based from the report by data.worldbank.org that Thailand's annual GDP per capita growth is $1.923 \%$, and web.nso.go.th states that working population amounts to $67.9 \%$ of total population. Also, the initial value of GDP per capita used to start the simulation is then estimated as 


$$
\begin{aligned}
b_{0} & =(\text { GDP before COVID })(1-\text { tourism input })(1-\text { GDP drop }) \\
& =\$ 7,792(1-0.13)(1-0.053)=\$ 6,419.75,
\end{aligned}
$$

taking the contribution from tourism to be $13 \%$, on the average, which is lost due to travel limitations during the lockdown period. The simulation starting week, $n=0$, corresponds to 3 weeks before lockdown was supposed to be first lifted in Thailand on April 29, 2020.

The initial value of weekly infection cases $i_{0}$ is taken to be 42 new cases which was the reported cases at 3 weeks before the lockdown was first lifted in Thailand, namely the week from April 22, 2020 to April 29, 2020.

Based on the daily reports of the Thai authorities on COVID-19 situation, in the month of April, the number of inbound travelers per week ranged from 1193 to 2661 [14]. In the past few months, there have been reports of 1 to 2 infected persons who were able to leave their quarantine centers and roamed free in the communities. From such reports, we can thus take the weekly increment in infected cases $v$ to be 0.00025 million in our simulations. Imposition of stricter quarantine measures will of course reduce this number.

The initial value of healthy mobile individuals $h_{0}$ is $h_{0}=($ fraction of population mobile $) \times($ initial population $)$

$$
=\left(1-q_{0}\right) \times 69.8 \text { million }=0.2 \times 69.8=13.96 \text { million } .
$$

Assuming that the lockdown has prevented interaction among the country's population to the level that only $20 \%$ or 0.2 of the total population of 69.8 million [15] remains mobile in the community.

We shall experiment with model simulations where the lock down is kept for 9 weeks, followed by a period of lockdown relaxation for 9 weeks. As realistically, businesses would need time before they can recover completely to their original capacities, we simulate the model with various delays $d$ in business recovery.

\section{B. Some Examples}

To show that reasonable parameter values exist that satisfy the conditions given in the Theorems in the previous section that ensure stability or instability of the equilibrium solutions of the model system, we give a couple of examples in illustration.

In the following examples, we let

$$
L=0.01, \beta=0.03, T=9, d=3 \text {. }
$$

Then,

$$
\frac{d \ln (1+\beta)}{\ln (1+\beta)+2 \ln (1-L)}=\frac{3 \ln 1.03}{\ln 1.03-2 \ln (1.01)}=9.164,
$$

and (11) holds.

Example 1: Suppose the fraction of population being put under lockdown is $q_{d k}=0.4$, then

$$
\frac{1}{1-q_{d k}}-\left(1+q_{d k}\right)=\frac{10}{6}-1.4<0.27 \text {. }
$$

Therefore, we may set

$$
\Delta=0.27>\frac{1}{1-q_{d k}}-\left(1+q_{d k}\right),
$$

and the fraction of population allowed out of lockdown is then

$$
q_{u k}=0.4+\Delta=0.67,
$$

in which case the steady state $\left(h_{s}, b_{s}, i_{s}\right)$ of $(1)-(6)$ will be unstable since

$$
\begin{aligned}
\lambda_{1} & =\left(1+q_{u k}\right)\left(1-q_{d k}\right)=\left(1+q_{d k}+\Delta\right)\left(1-q_{d k}\right) \\
& >\left(1+q_{d k}+\frac{1}{1-q_{d k}}-\left(1+q_{d k}\right)\right)\left(1-q_{d k}\right)=1,
\end{aligned}
$$

which is the desirable outcome in which the mobile population does not become extinct.

On the other hand, if we set

$$
\Delta=0.1<\frac{1}{1-q_{d k}}-\left(1+q_{d k}\right),
$$

and the fraction of population allowed out of lockdown is then

$$
q_{u k}=0.4+\Delta=0.5,
$$

in which case the steady state $\left(h_{s}, b_{s}, i_{s}\right)$ of $(1)-(6)$ will be stable, which is an undesirable scenario as the mobile healthy population eventually becomes extinct, while the infection becomes endemic.

For the model system (16) - (21), we consider the following examples.

Example 2: If $L=0.00663, \beta=0.03, q_{d k}=0.8, q_{u k}=0.2, T=$ $9, d=3$, then

$\frac{d \ln (1+\beta)}{\ln (1+\beta)+2 \ln (1-L)}=\frac{3 \ln 1.03}{\ln 1.03-2 \ln (1.00667425)}=5.4751$

and

$$
\frac{1}{\ln \left(1+q_{u k}\right)} \ln \left(\frac{1}{1-q_{d k}}\right)=8.82747
$$

Thus, we obtain

$$
T_{m}=\min \{8.82747,5.4751\}=5.4751<9=T .
$$

Hence, according to Theorem $2,\left(h_{s}, b_{s}, i_{s}\right)$ is unstable.

Example 3:

$$
\begin{aligned}
& \text { If } q_{d k}=0.8, q_{u k}=0.15 \text {, then } \\
& \qquad \frac{d \ln (1+\beta)}{\ln (1+\beta)+2 \ln (1-L)}=\frac{3 \ln 1.03}{\ln 1.03-2 \ln (1.01)}=9.164
\end{aligned}
$$

and

$$
\frac{1}{\ln \left(1+q_{u k}\right)} \ln \left(\frac{1}{1-q_{d k}}\right)=\frac{\ln 5}{\ln (1.15)}=11.51 .
$$

We thus obtain

$$
T_{m}=\min \{11.51,9.164\}=9.164>9=T .
$$

Hence, according to Theorem $2,\left(h_{s}, b_{s}, i_{s}\right)$ is stable.

\section{Model Simulation}

Simulations of the model have been carried out specifically to try to answer some pressing and difficult questions that the authorities of many countries are facing and finding hard to answer in order to make the right decision concerning whether a lockdown should be imposed and how it is to be carried out. We therefore vary the model's parameters to illustrate various scenarios in which different strategies are adopted with different outcomes.

In the following figures, we show graphs of the state 
variables of interest as functions of the number $n$ of weeks beyond the start of the simulation when $n=0$, corresponding to 3 weeks before the first lockdown is lifted in Thailand.

In Fig. 1, the system (1) - (6), which models abrupt opening of businesses, with $q_{u k}=4.0, q_{d k}=0.8$, is simulated with period of lockdown $T=9$ weeks, the delay in business recovery $d=3$ weeks, portion of businesses connected to visitors and workers from abroad is $\beta=15 \%$ of the current GDP, but they are allowed in every 9 weeks. The number of new infected cases is incremented from the infected travelers from abroad by $v=2.5 \times 10^{-4}$ millions each week during $(2 k+1) T+d$ and $(2 k+2) T, k=1,2,3, \ldots$.

Number of new cases

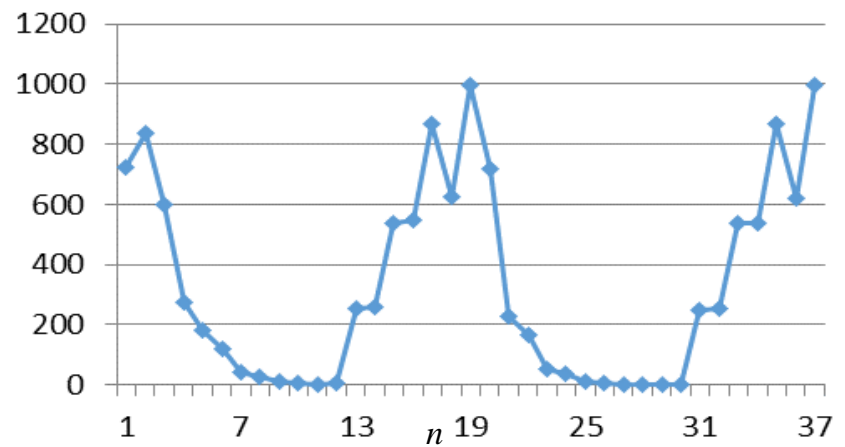

a)

GDP per capita

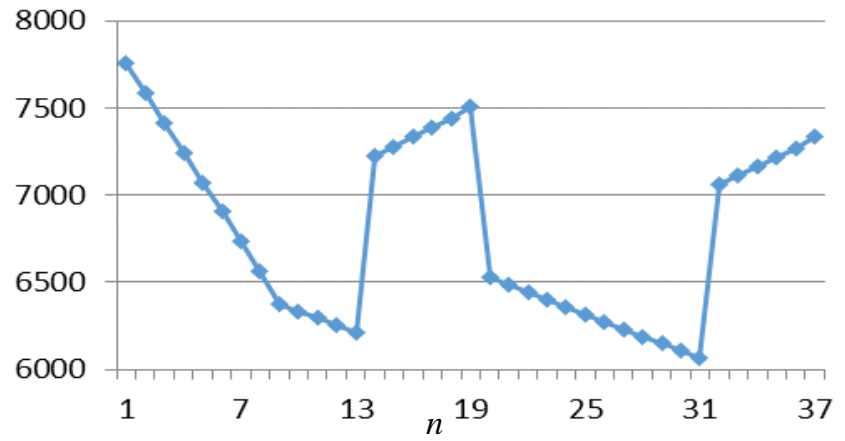

b)

Fig. 1 Simulation of system (1) - (6) for the case where lockdown is lifted in an abrupt manner and the delay $d$ is 3 weeks: a) number of weekly new cases, b) GDP per capita. The simulation starts from the point where $n=7$ on the graph.

In Fig. 1, we show the data of infected cases and national GDP per capita 6 weeks before the simulation is started in order to allow the readers to compare the situations before the crises and after it actually showed signs of serious repercussions on the economy. We see in Fig. 1a) that the GDP level is not able to recover its original level 9 weeks after the lockdown has been lifted. Also, when the alarming rise in infected case, seen in Fig. 1a), forces the second lockdown after 9 weeks, the level of GDP per capita continues to drop, showing no sign of possible future recovery provided that this condition remains unchecked. This is because business recovery takes 3 weeks at least to take place if there were no assistance from the government to stimulate the economy in a big way.

We therefore look at the scenario in which the government hands down a huge stimulating package to help the economy recover so that the time required for it to recover is shorter, by putting $d=1$. The model simulation in this scenario is seen in Fig. 2. where $q_{u k}=4.0, q_{d k}=0.8$ and $\beta=15 \%$ as well.

Number of new cases

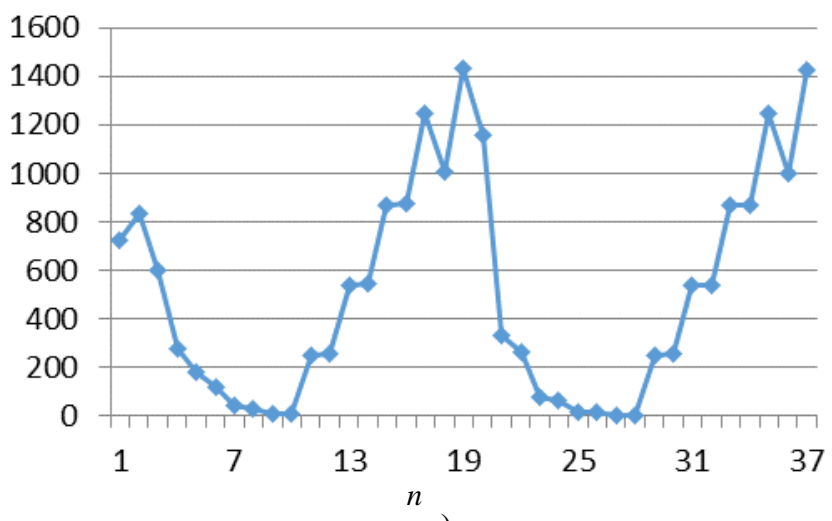

a)

GDP per capita

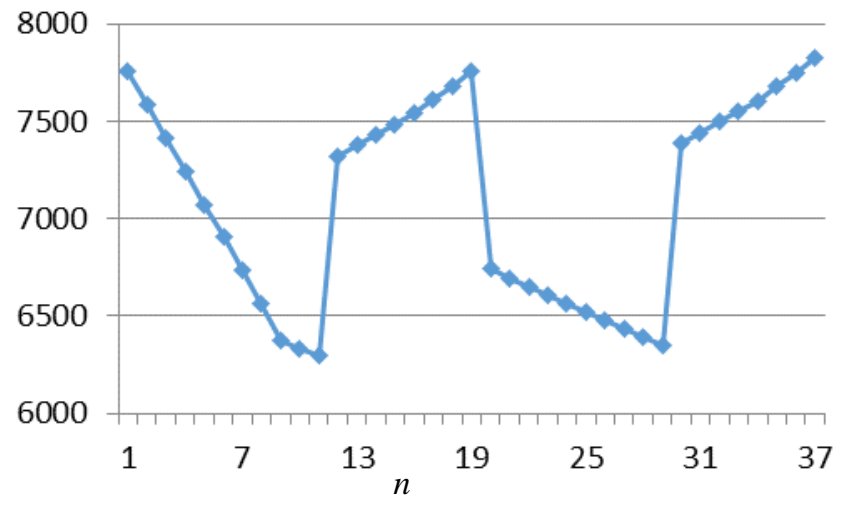

b)

Fig. 2 Simulation of system (1) - (6) for the case where lockdown is lifted in an abrupt manner and the delay $d$ is 1 week: a) number of weekly new cases, b) GDP per capita. The simulation starts from the point where $\mathrm{n}=7$ on the graph.

We see in Fig. 2b) that in this case where firms and businesses can recover more quickly, the national GDP is able to recover its original value after 9 weeks after the lockdown has been lifted. This appears to be a satisfactory strategy that the government might follow. However, we observe in Fig. 2a) that the number of weekly new cases rises a much higher level which might overwhelm the public health system. This is most likely due to the abrupt lifting of the lockdown from which the population become mobile all at once, or no social distancing and mask wearing are not observed to a satisfactory extent. We therefore carried out a simulation of the model system (16) (21), shown in Fig. 3, which simulates a scenario where the 
lockdown is lifted in an incremental fashion. This may be thought of as stricter observation of masks usage and social distancing even when the lockdown is lifted. The simulation result in this scenario is shown in Fig. 3 where healthy mobile population is incremented by $q_{u k}=0.198$ repeated from $n=(2 k-1) T$ to $(2 k+1) T, k=1,2,3, \ldots$, in a gradual fashion.

Number of new cases

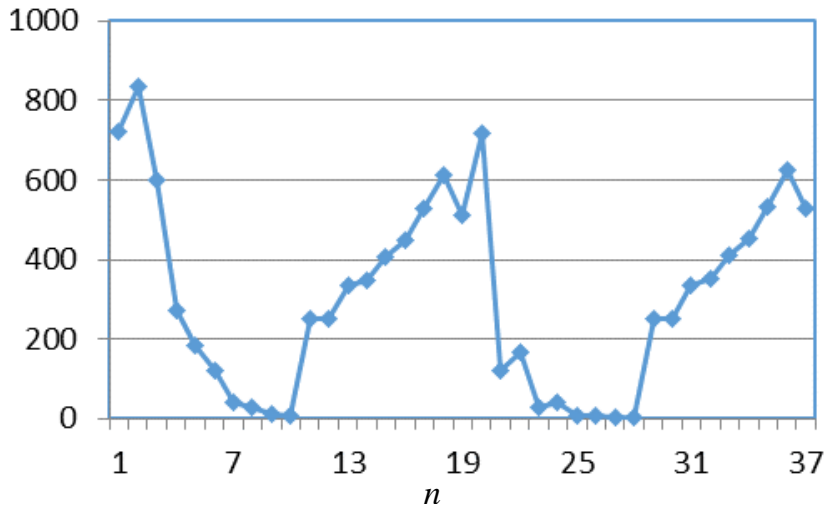

a)

GDP per capita

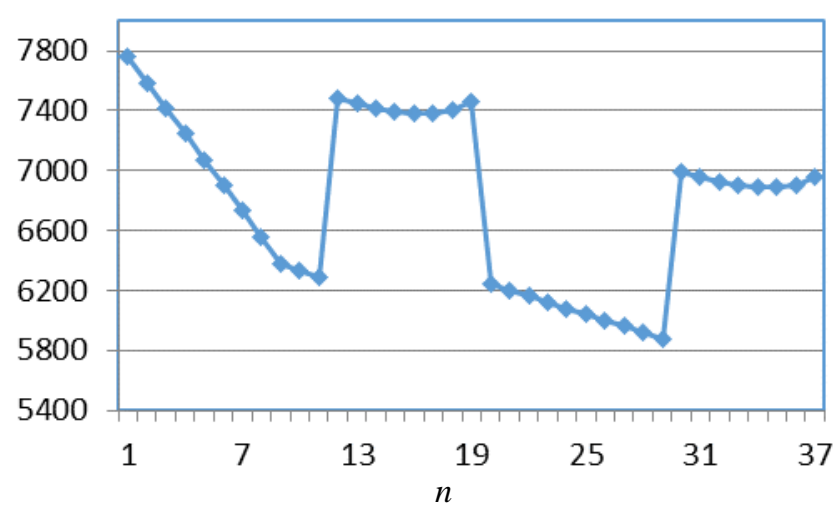

b)

Fig. 3 Simulation of system (16) - (21) for the case where lockdown is lifted in an incremental manner and the delay $d$ is 1 week: a) number of weekly new cases, b) GDP per capita. The simulation starts from the point where $\mathrm{n}=7$ on the graph.

We observe in Fig. 3 that the number of new cases each week is now lower which could be more manageable. However, as expected, the economic recover becomes less satisfactory. This is due to the fact that mobile individuals become active in the interaction with each other in a gradual fashion. Social distancing may be keeping them apart or not get involved in certain risky transactions. Businesses then do not recover fast enough.

Experimenting with shorter lockdown periods does not yield much better recovery. Based on these model simulations, therefore, we suggest a strategy that involves an incremental relaxation, but with a bigger jump at the week right after the lockdown ends, followed by smaller increments in a reasonable and realistic manner. Fig. 4 shows the simulation in this case with the following incremental rule instead of that in (19):

$h_{n+1}=\left\{\begin{array}{l}\left(1+q_{u k 1}\right) h_{n}, \text { if } n=(2 k-1) T \\ \left(1+q_{u k 2}\right) h_{n}, \text { if } n=(2 k-1) T+m, m=1,2,3 \ldots, T-1 . \\ \left(1-q_{d k}\right) h_{n}, \text { if } n=2 k T\end{array}\right.$

Number of new cases

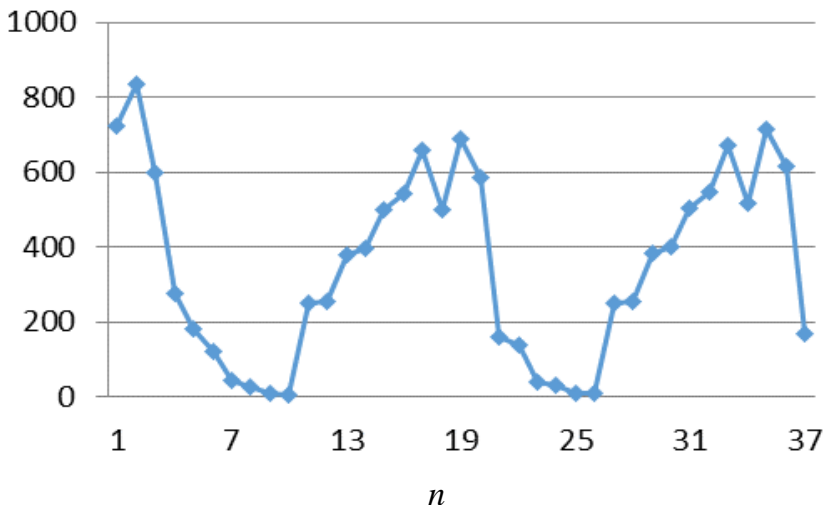

a)

GDP per capita

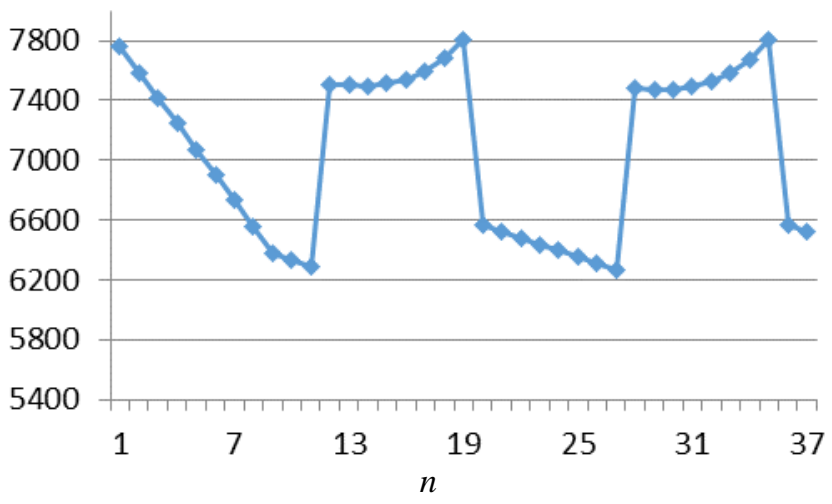

b)

Fig. 4 Simulation of system (16) - (21), with (19) replaced by (28), for the case where lockdown is lifted in an incremental manner and the delay $d$ is 1 week: a) number of weekly new cases, b) GDP per capita. The simulation starts from the point where $\mathrm{n}=7$ on the graph. The second lockdown lasts only 7 weeks.

In Fig. 4, $q_{u k 1}=1.0$ and $q_{u k}=0.124$. We see that, if we adopt this rule in (29), as well as a shorter lockdown, the economy recovery is very promising, as seen in Fig. $4 \mathrm{~b}$ ), while number of new cases does not peak at a very high level. In fact, the peak here is more or less the same as in Fig. 3a), and it might be still manageable with respect to the health care capacity. If we take the business recovery to be an important factor in decision making, this option appears to be a good strategy to follow. In terms of infected cases, the difference between strategy shown in Fig. 3 and that seen in Fig. 4 would be in the cummulative number of infected cases, seen in Fig. 5.

Cumulative number of cases 


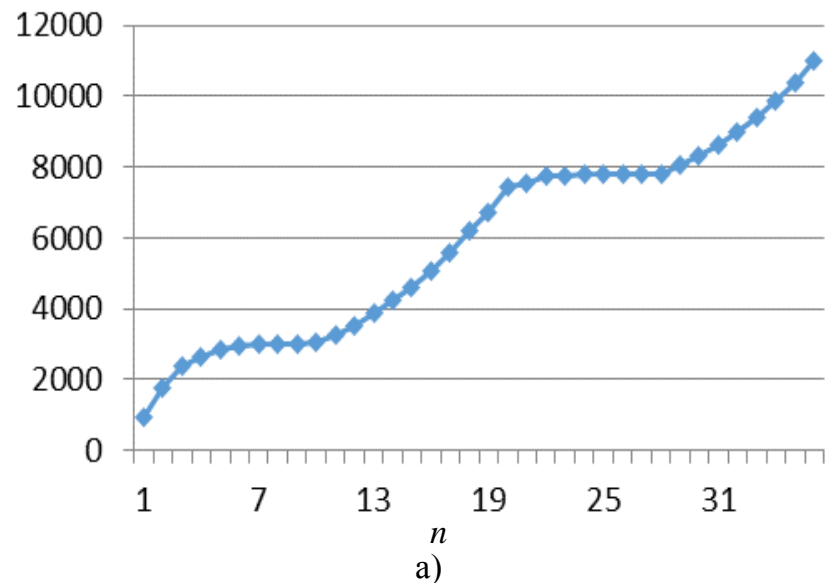

Cumulative number of cases

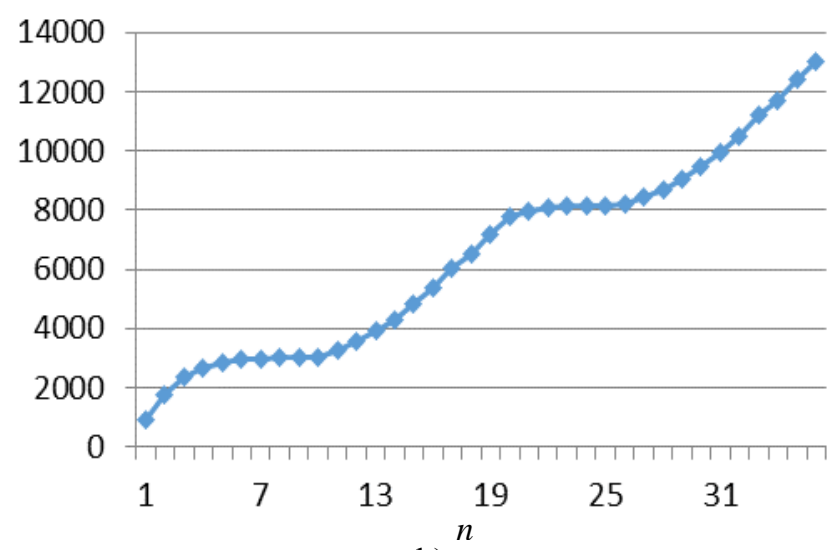

b)

Fig. 5 Simulated weekly cumulative cases corresponding to a) strategy seen in Fig. 3, b) strategy seen in Fig. 4.

We see in Fig. 5, which compares the strategies simulated in Fig. 3 and Fig. 4, the cumulative number of infected individuals resulting from the strategy proposed for Fig. 4 is higher than that for Fig. 4. This is because the population comes out of lockdown as a large increment in $h_{n}$ on the first week and greater numbers of new cases are recorded which increases the cumulative number earlier on. Also, the lockdown does not last very long in Fig. 4, only 7 instead of 9 weeks which leads to a higher cumulative number.

A further simulation carried out on (16) - (21) illustrates the outcome when the lockdown is carried out in a gradual fashion. Since the infection rate, seen in Fig. 6a), is not down to a low enough level, the lockdown process has to be kept longer before the number of new cases is in the single digits. This then hurts the economy more as seen in Figure $6 \mathrm{~b}$ ), where $q_{d k}=0.172$ every week for 12 weeks.

Number of new cases

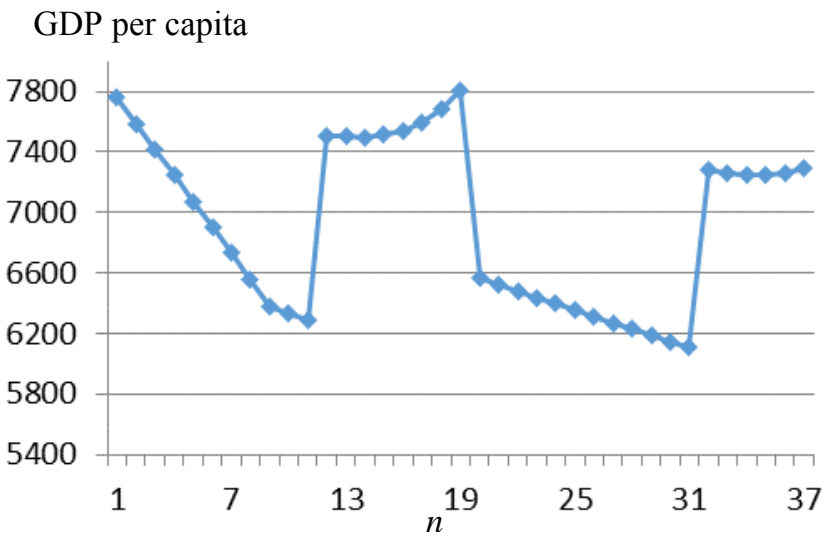

b)

Cumulative number of cases

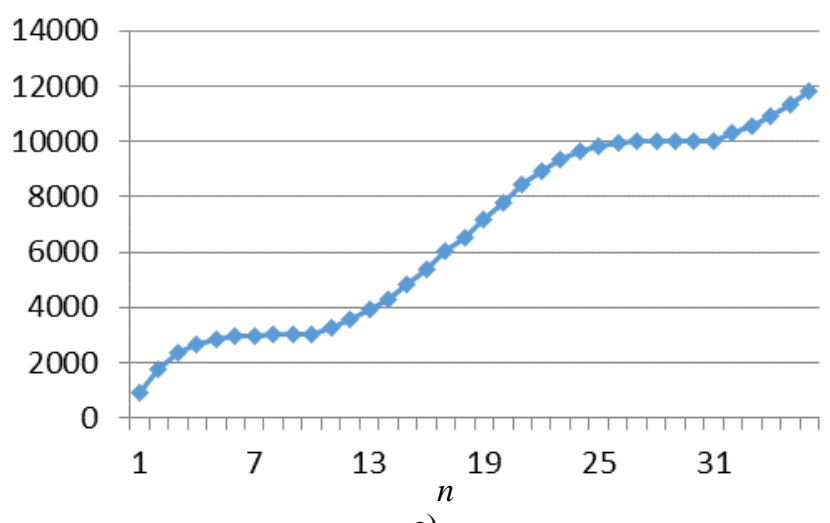

c)

Fig. 6 Simulation of a modified model in the case that the lockdown is gradual, stretching 12 weeks as described in the text: a) number of new cases, b) GDP per capita, and c) cumulative number of cases.

Even though $q_{u k}=2.8$ first week into the uplift of lockdown followed by 0.124 as before on later weeks as in Fig. 5, it does not help the recovery second time around that much. The cumulative number seen in Fig. 6c) does not appear higher than those in Fig. 5 at the end of the simulation, but this is because the second opening up of lockdown is not through yet at $n=37$. The cumulative number will continue to rise to a level higher than the other 2 scenarios before lockdown has to be imposed again after 9 weeks. 


\section{CONCLUSION}

We have proposed and analyzed models that describe the interaction between COVID-19 infection in a community and the health of its economy. The models allow us to investigate the effect of the spread of corona virus infection and the consequent measures taken in response to its spread to lessen its impacts on the society and the economy. Impulses in healthy mobile population and the national GDP level due to the lockdown impositions and relaxations are incorporated. Moreover, there are 2 delays in our models. We assume that an infected individual takes an average of 2 weeks before being detected. The role of recovered individuals is also taken to account in the rate of increase of GDP with a delay of 4 weeks on the average before the recovered individuals may return to the mobile population. Thus, our models become an impulsive system of difference equations with multiple delays.

To highlight the conclusions reached by the application of our models, it is clearly illustrated that sharp lockdowns and sharp opening up of lockdowns would yield more favorable outcomes, both in terms of economic recovery and control of the infection rate. A gradual lockdown brings the infection rate down more slowly. Also, the lockdown has to be kept longer before the infection rate gets satisfactorily low, which means the economy is hurt for a longer period since it cannot open up for business. Thus, it is deemed more prudent to impose a sharp lockdown to quickly bring down the number of cases so that the lockdown can be relaxed sooner. On the other hand, it has been shown by our model simulations that, while a gradual relaxing of lockdown can be a good measure to curb the rise of infection rate, it is detrimental to the recovery of the economy. A combination of sharp and incremental relaxations is recommended to simultaneously control the infection rate and stimulate quick economic recovery.

Thus, the models provide us with a qualitative projection of the outcomes of different strategies in lifting the lockdowns or mandating them, which can be very valuable for the decision makers in trying to navigate the difficult task of balancing between keeping the infection rate low and pushing for economic recovery. In making such decisions, qualitative projections are extremely important since they answer the questions of "what if", also explaining the causes and effects of the outcomes, better than a model that fits a set of data closely but does not describe the dynamic relationships between different important factors at play in the phenomena of interest, the understanding of which is crucial if we were to know what would entail if a factor is changed, and why, including which factors should be adjusted in our strategies in order to affect the desirable outcome.

\section{ACKNOWLEDGMENT}

Appreciation is extended toward Mahidol University, and the Centre of Excellence in Mathematics, CHE, Thailand, Post Graduate Education and Research Development Office of the Ministry of Higher Education, Science, Research, and Innovation, Thailand.

\section{REFERENCES}

[1] D. Bachman, "The economic impact of COVID-19 (novel coronavirus)" Deloitte Insights, March 3, 2020. Available: https://www2.deloitte.com/ us/en/insights/economy/covid-19/economicimpact-covid-19.html.

[2] P. Carlsson-Szlezak, M. Reeves, and P. Swartz, "Understanding the Economic Shock of Coronavirus, Howard Business Review," Economics \& Society, March 27, 2020. Available: https:/hbr.org/2020/03/ understanding-the-economic-shock-ofcoronavirus.

[3] D. M. Thomas, R. Sturdivant, N. V. Dhurandhar, S Debroy, and N. Clark, "A Primer on COVID- 19 Mathematical Models," Obesity, Wiley Online Library, vol. 28, Issue 8, 09 May 2020.

[4] W. O. Kermack, and G. McKendrick, "A contribution to the mathematical theory of epidemics," Proc. R. Soc. Lond., A 1927, pp.700- 721 .

[5] N. P. Jewell, J. A. Lewnard, and B. I. Jewell, "Predictive mathematical models of the COVID- 19 pandemic: underlying principles and value of projections," JAMA, April 16, 2020. Available: doi:10.1001/jama.2020. $\underline{6585}$.

[6] Y. Liu, A. A. Gayle, A. Wilder- Smith, and J. Rocklov, "The reproductive number of COVID- 19 is higher compared to SARS coronavirus," J. Travel Med., 2020, vol. 27. Available: doi: $10.1093 / \mathrm{jtm} / \mathrm{taaa} 021$.

[7] D. Champredon, J. Dushoff, and D. Earn, "Equivalence of the Erlang- distributed SEIR epidemic model and the renewal equation," SIAM J. Appl. Math., 2018, vol. 78, pp. 3258- 3278.

[8] E. Massad, M. N. Burattini, L. F. Lopez, and F. A. Coutinho, "Forecasting versus projection models in epidemiology: the case of the SARS epide-mics," Med Hypotheses, 2005, vol. 65, pp. 17- 22.

[9] “Covid-19 Situation Reports," Department of Disease Control, Ministry of Public Health. Available: https://covid19.ddc.moph.go.th/en

[10] World Population Review. Available: https://worldpopulationreview. com/countries/thailand-population.

[11] "Thailand - Gross Domestic Product Per Capita in Current Prices," World Data ATLAS, KNOEMA. Available: http://KNOEMA.com/ATLAS/ Thailand/ GDP-per-capita.

[12] Tourism in Thailand, Wikipedia. Available: https://en.wikipedia.org/wiki/ Tourism_in_Thailand.

[13] "Daily Strategy for Investors," Kaohoon Online. March 26, 2020. Available: https:// www.kaohoon.com/content/350411.

[14] "COVID-19", Department of Disease Control, Ministry of Public Health. Available: https://ddc.moph.go.th/viralpneumonia/situation.php

[15] "Capital Facts for Bangkok, Thailand," World's Capital Cities. Available: $\quad$ https://www.worldcapitalcities.com/capital-facts-forbangkok-thailand/.

[16] S. Raimo, "Qualitative VS. Quantitative Research," Scribbr. April 12, 2019. Available: https://www.scribbr.com/methodology/qualitativequantitative-research/.

\section{Creative Commons Attribution License 4.0 (Attribution 4.0 International, CC BY 4.0)}

\author{
This article is published under the terms of the Creative \\ Commons Attribution License 4.0 \\ https://creativecommons.org/licenses/by/4.0/deed.en US
}

